\title{
How do agent types represent human behaviour in land use change modelling?
}

\author{
$\underline{\text { H. van Delden }}{ }^{\text {a }}$ and J. van Vliet ${ }^{\text {a }}$ \\ ${ }^{a}$ Research Institute for Knowledge Systems, Witmakersstraat 10, 6211 JB Maastricht, The Netherlands \\ Email:hvdelden@riks.nl
}

Land use change models are frequently used as decision support systems, tools to explore possible future developments of a city, region or country. Given that spatial developments are to a large extent driven by human action and interaction, agent based approaches are a logical choice for simulating these processes, as agents are a natural representation of actors that make decisions. Over the past decade, several prototypes and more advanced simulation models have been reported that claim to include agents, or at least include agent behaviour. When analysing these different models, it quickly becomes clear that the definition of agent based in land use change modelling is rather broad and varies in the way decision-making bodies are perceived and represented in the model.

Based on our analysis we found that agent based land use models can be categorised in five main types, which can be ordered on a continuum from a more abstract representation of agents to a purer and narrower definition of agent based modelling: (1) cellular automata models, (2) activity or density based models, (3) models with cellular agents, (4) models with global agents, and (5) models with local agents. While this typology might not be exhaustive, most agent based land use models that are currently available can be placed in one of these categories.

In this paper we describe these five types of agent based land use models in more detail, focussing on the way they represent human behaviour and human decision making and the consequences this has for simulating land use developments. Specifically, the overview examines what types of decisions an agent type can make, the scale at which decisions take place, and the detail of the processes represented. Furthermore, we analyse each type in terms of their practical applicability in spatial decision support systems, such as the data and computation requirements for model applications, the temporal and spatial scale at which each of these models can be applied, the possibilities for calibration and validation and the type of information that they obtain.

We found that all five types incorporate human behaviour and human decision making in some way, but that the degree to which they do and the detail with which they do so varies considerably. At the same time we found that detail in agent representation generally comes at the cost of data and computation requirements as well as an increase in uncertainty. Cellular automata models are the least demanding in terms of data and computation power, consequently they represent human behaviour in their most abstract form. Models with local agents on the other hand generally have the most detail in the way human decision making is represented as they allow to represent each individual, family or farm explicitly including their relation to space. However, this detail comes at the cost of the data requirements and difficulties in calibration and validation. The other three types are in between these two extremes.

We conclude that there is not one best way to include agents in spatial decision support systems, but that instead this decision is a trade off between the different properties related to each of the five types. The decision on the type of agent behaviour to be incorporated in a spatial decision support system highly depends on data availability and area extent, but also on the type of information and practical considerations required by the (potential) users.

Keywords: Behavioural modelling, Agent based modelling; Cellular Automata; Land use modelling; Spatial Decision Support System. 


\section{INTRODUCTION}

Land use models have been used to study the changes in land use developments, and increasingly to perform scenario studies and analyse policy alternatives (Sieber et al., 2010). To meet the requirement for policy impact assessment, these models are often incorporated in spatial decision support systems (SDSS) that enable what-if type of analysis and hence incorporate causal relationships.

Land use changes, and in particular those that are caused or affected by policy measures, are to a large extent driven by human decisions: farmers decide which crop to grow, governments choose to protect pristine areas and families select a place to live. For a meaningful assessment of the influence of external drivers as well as policy options on land use changes, it is therefore required to represent human behaviour and human decision making in these models. The way in which this behaviour and decision making is included in land use change models is often referred to as agent based modelling. Agent based here refers to the idea that the decision making agent is represented explicitly or implicitly in the model. A closer look at these models however tells us that the implementation of these agents, their properties, their relation to space and the way they can make decisions differs considerably for the various types of models. Related to this we find that the practical aspects of these models, like the data requirements and the domain of applicability, show large variations.

Several overviews on agent based models (ABM) exist. For example Matthews et al. (2007) discuss various models according to their purpose and Robinson et al. (2007) discuss alternative approaches to ground ABM empirically. In this paper we try to identify and characterize the different types of agent based models that are available for land use change modelling. We do so according to two main points of analysis. First, we focus on the behaviour and decision making of spatial agents represented in the system, including the relation between the agent and space. Second, we assess for each type of model the practical issues in relation to scenario studies and spatial decision support. While doing so we do not aim to judge or rank these models, instead we intend to show that there is a broad range of agent based models available and try to categorize them.

We start in section two of this paper with a description of the five types of agent based models that we identified in this overview. Section three subsequently compares these types of agent based models based on the two focus points mentioned above. Section four then draws some conclusions on the use of these models for scenario studies and for applying them in spatial decision support systems.

\section{APPROACHES FOR INCLUDING HUMAN BEHAVIOUR IN LAND USE MODELLING}

In the following section we will present the characteristics of the five categories of agent based land use models found in literature, ranging from models with a more abstract representation of agents to a purer and narrower definition of ABM: (1) cellular automata models, (2) activity or density based models, (3) models with cellular agents, (4) models with global agents, and (5) models with local agents.

\subsection{Cellular Automata}

Cellular automata (CA) land use models exist on a lattice of cells. Each cell on the lattice has one of a limited number of cell states which represents the predominant land use at that location. In each discrete time step, cell states are updated simultaneously according to a set of transition rules. The characteristic of CA models is that the state of adjacent cells, and hence the land use in the neighbourhood of a location, is input to the transition rules. Additional factors such as infrastructure, physical aptness or policy regulations are often added to represent heterogeneous geographic features. One can distinguish between constrained CA models in which land use demands are determined exogenously and only the allocation is simulated through the modelling approach, and unconstrained CA models in which the land use of a location is strictly the result of local characteristics.

Agent behaviour in CA is represented in the parameterization of the various drivers, most notably the neighbourhood effect. The transition rules underlying the neighbourhood effect can,

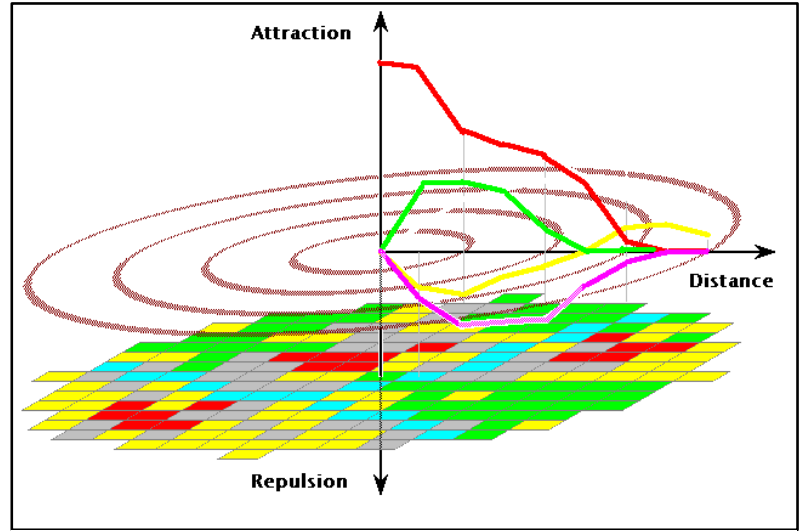

Figure 1. Schematic representation of the incorporation of human behaviour in CA models through transition rules for land use change. 
obviously, differ from one land use class to another indicating that the actors associated with this land use have different preferences. For example industrial areas might prefer a location close to the motorway exits, while that is less important for residential land uses. Together the transaction rules describe the allocation preferences of the actors related to the different land uses as well as their economic and political power to actually occupy the preferred locations. For constrained land uses this implies that agent behaviour is represented on the level of the land use classes. After all, the particular land use type is looking for a location to place itself. In the case of unconstrained land use states the agent behaviour is represented at the cellular level. As opposed to constrained land uses there is not one land use looking for a number of locations, but a cell deciding what land use to take. As the latter usually refers to natural land uses, this behaviour is hardly related to agents though. Hence, in most CA models, the land use is the agent, who searches for the optimal location for his activity. In that process he can face the competition of other agents (land-use types) that are also looking for a suitable location. Heterogeneity in agent behaviour is included by selecting a set of land use classes that are perceived to change over time differently due to the behaviour of related actors. For example the land use class 'low income residential' may have a different behaviour than 'high income residential', which is included in the transition rules of the related class. Differences in individual preferences are often incorporated using a random factor in the allocation.

Some well known examples of CA land use models are SLEUTH (Clarke et al., 1998) and Metronamica (RIKS, 2010). Both models have been applied in areas ranging from cities to continental scales. Because of their rather limited data requirements CA based applications have been set up all around the world and various applications have been calibrated against historic data. Their limited data requirements, stylistic process representation and related fast computation speeds have stimulated their use in SDSS. An example of studies in which CA models are used in planning processes is provided by De Nijs et al. (2004).

\subsection{Activity Based}

Defining 'the' land use on a location is often hard to do, if not impossible. Mostly we find a mix of land uses and activities at a particular location: in city centres commercial activities are often mixed with residential dwellings, infrastructure and public services and in the countryside one can find a mixture of agriculture, farm houses, industry, recreation, etc. Furthermore, land use changes are typically not exhibited in sudden changes of the predominant land use, but instead by gradual changes in a mix of activities that is taking place within a certain area.

Recently, cellular automata models have been expanded with more detailed information on the activities that are taking place on a location, such as its number of inhabitants or the number of jobs. This addition to the CA framework is named activity based modelling. Activity based modelling bears large resemblance to the CA models described above, but adds to this the density information: activity based models exist on a raster of grid cells, and each cell typically has a predominant land use and a density of the associated activity or a vector of activities present at that location. Land uses and activity densities change in discrete time steps according to a set of transition rules that typically include a neighbourhood effect and other characteristics related to the geographical space.

Agent behaviour in activity based models is represented through the transition rules. In constrained activity based models agent behaviour is effectively represented at the level of the activity. Generally the activity is allocated on the lattice and the associated land uses are subsequently allocated according to the activity

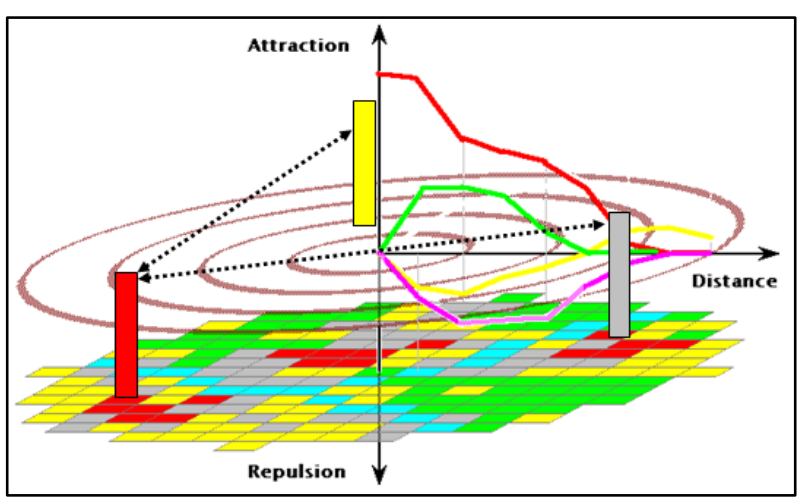

Figure 2. Schematic representation of the incorporation of human behaviour in activity based models by including activities per cell in addition to the land use of the location. distribution. As the activity does not necessarily need to relate to the predominant land use on a location and a location can have more than one activity, this approach does allow for mixed land uses. This modelling approach allows for heterogeneity in actor behaviour through the selection of the activities and the parameterisation of the transition rules. Individual actors are not represented as such but instead as a density unit of their activity type. Differences in individual preferences are often included through a random factor in the allocation.

Examples of activity based models are presented in Yeh and Li (2002) and Van Vliet et al. (2011). Their scale varies from cities to countries. All current applications seem to be in a research phase 
and data for calibrating densities at cellular level appears to be a limiting factor in a historic calibration. We have not been able to find any evidence of the use of activity based models in SDSS nor in spatial planning exercises.

\subsection{Models with cellular agents}

As the name implies, models with cellular agents include cells that act as an agent. These models exist on a lattice of cells, and cells generally have a state, representing the land use of the location. These states and especially the mechanisms according to which they change normally include quite a bit of detail on the agent and his behaviour through the agent's attributes, beliefs, desires, intentions, and decision-making mechanisms.

In these models cells are the decision makers through which actor behaviour is expressed. Typically the cell is supposed to represent an agent who has a direct relation to the land, such as a farmer or owner. Decisions therefore directly

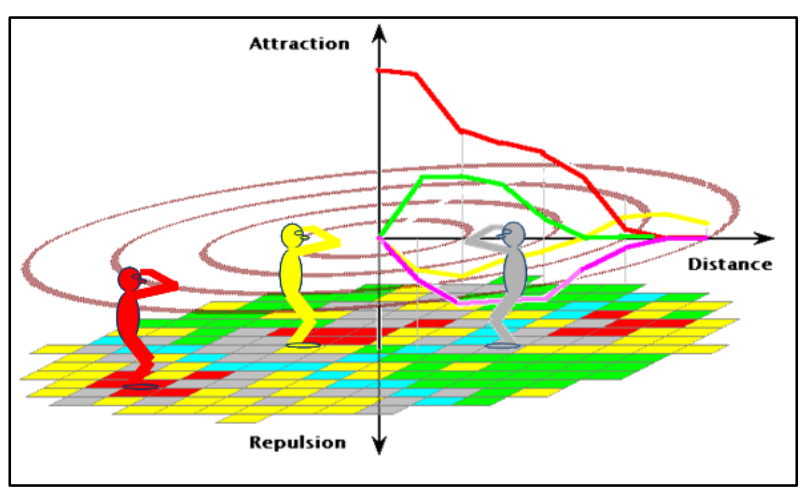

Figure 3. Schematic representation of the incorporation of human behaviour in cellular agent models in which agents are represented by cells. relate to the location that is represented by the grid cell, such as decisions on which crop to farm or whether to develop or not. Their link to a specific location limits their behaviour as agents cannot freely move in the modelled area. Another limitation is their explicit link to a certain area extent. In the case of agricultural applications this means that it is assumed that a farmer makes a decision on each plot (a cell) independently of his other plots (other cells), as the relation between his plots is not specified in such applications. The type of information provided by these models is normally wider than just the land use on a plot and can for example include information on yields, agricultural profits or land prices.

An example of this type of model where cells represent farmers is presented in Van Delden et al. (2007). Due to the detail that is included in the decision-making process, models that include cellular agents are typically applied on a smaller scale, such as a watershed or a city. We have not yet been able to find real-world applications showing a historic calibration. There is mention of these models as part of SDSS (Van Delden et al. (2007) as well as their use in scenario studies (Kok and Van Delden, 2009).

\subsection{Agent based with global agents}

The global agent based approach follows a two-step procedure to simulate land use changes. On the global level, not strictly connected to space, there are agents who interact and compete. These agents can represent groups of spatial actors, such as environmentalists, project developers or farmers. The outcome of the agent interaction at the global level is used as one of the drivers of land use change at local level. For example the result of a political process representative for the study region determines whether an area represented by local cells is allowed for development or whether it is preserved as a natural area. At local level these models tend to have a grid space where cells have a land use state, although this is not necessarily required and polygon-based allocation approaches could also be used. The approach in itself does not require a specific spatial configuration.

The agents in this approach are represented as groups that have an influence on land use dynamics. Agent behaviour and interaction between agents does not

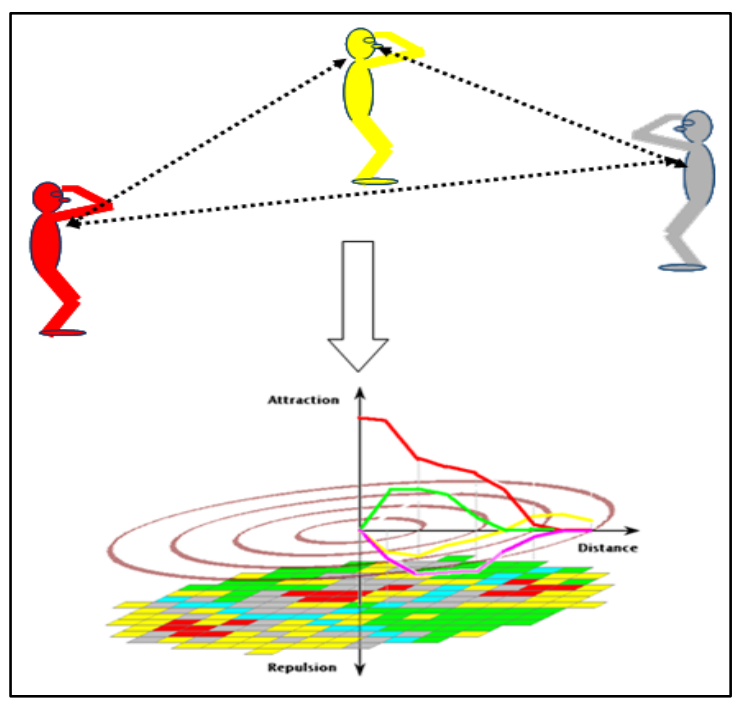

Figure 4. Schematic representation of the incorporation of human behaviour in agent based models with global agents whom interact globally and whose global decisions then impact locally. take place at the local level and there is no relation between an agent and a particular location. Interaction between agents is therefore inherently not spatial but economic or political. As the agent represents a larger group these models look at the desires, believes and intentions of groups, rather than those of any individual. 
Therefore these agents are particularly suitable to simulate top-down effects that are the result of an economic or political discussion.

An example of this type of model is a series of studies presented by Ligtenberg et al. (2001). They simulate land use changes in a small rural region in the Netherlands. Other approaches found (e.g. www.plurel.net) are also applied at the regional scale. So far we have not yet seen these models as parts of SDSS. Agent based models with global agents can include quite a bit of detail on the agent behaviour, but as the agents are large groups of people rather than individuals data requirements seem to be moderate. As interaction between agents is normally limited to interaction between a few groups, computational requirements tend to be rather low, but of course are also influenced by the allocation procedure used.

\subsection{Agent based with local agents}

In agent based models with local agents, land use changes as the result of decisions taken by the agents at local level. These decisions can take into consideration both local drivers, such as the characteristics of a location and other land use in the vicinity, but also global processes, such as market prices or climate change. Eventually some global processes could emerge again from the combined effect of local agents.

In this approach the agent himself is the unit that makes decisions based on his on desires, believes and intentions and the interaction with other agents and his environment that result from this. Agents exist locally, which means they have an explicit relation to the space they populate. This relation can have the form of an agent owning a parcel of land and therefore has the ability to decide on and change his land use, or an agent representing a family that decides to live in a certain location or a farmer deciding which crops to farm. In agent based models with local agents, agents are not fixed to one location and are able to move freely in space. Moreover an agent can own and/or make decisions on a particular area, which can be a cell, a cluster of cells or a polygon. It is even possible for several agents to be situated on one particular location. Hence there is a separation from the agents and locations through different layers of the model.

The individual representation of agents and the amount of detail in these models has the obvious benefit that human behaviour can be approached closely. This however comes at the costs of computation time and data requirements. Consequently, as some overviews show (e.g. Parker et al., 2003), these models are typically only applied on relatively small scales, and with a limited number of agents, although exceptions do exist, see e.g. Smajgl, 2010. Some agent based models use synthetic agents to study land use change processes as data on individuals is not available or protected due to privacy restrictions. Examples of agent based models with local agents include the studies of Anasazi settlements in Longhouse Valley (Dean et al., 2000) and the work of Haase et al. (2010) studying urban environments. The use of this type of agent based models in SDSS seems limited, which might be due to the fact that most developers indicate that their model is intended to understand processes, rather than to provide accurate forecasts on future land use developments.

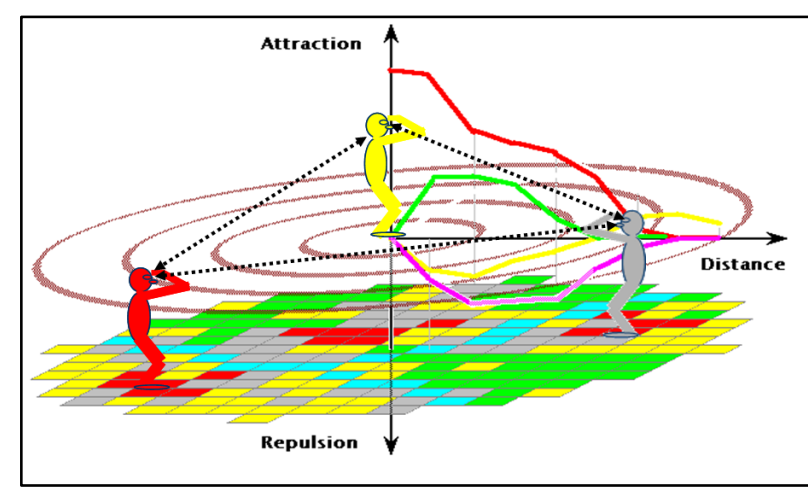

Figure 5. Schematic representation of the incorporation of human behaviour in agent based models with local agents whom can freely move and interact throughout the modelled area.

\section{COMPARISON AND DISCUSSION}

Comparing the models currently developed within the various categories (Table 1) we see that all approaches include human behaviour, but differences occur in the way this is represented. A main difference is the decision unit and the level of abstraction with which human behaviour is incorporated. Simulating each individual as an agent, the local agent based approach, is the closest one can come to resemble the process of human decision making. In this approach each agent can have his own characteristics, including his own believes, desires and intentions. Moreover, local agents allow including networks between agents explicitly. CA models, on the other extreme, have a rather abstract process representation in which agents are not included as such, but in which their behaviour is expressed through the transition rules for land use change.

In terms of complexity activity based modelling tries to find a middle ground between CA and local agent based modelling by adding additional information of activities to the CA and thus making both the results richer and the processes more realistic, without the need for an elaborate amount of data. Global agent based 
Van Delden and Van Vliet, How do agent types represent human behaviour in land use change modelling?

modelling is complex in its agent behaviour, but omits the local competition as well as the interaction of the agent with the environment at local level. Having the agent interaction at the level of the entire study area makes these systems less computationally intensive than the local variant. The complexity of the cellular agent approach comes close to the local agent based approach, but omits the separation between the agents and the landscape and therefore the free movement in space.

We have separated heterogeneity in human behaviour in two aspects. Firstly, the inclusion of different agent types with different behaviour and secondly the inclusion of individual differences within agent groups. Global, cellular and local agent-based models often work with an agent typology for various groups, while $\mathrm{CA}$ and activity based models include a differentiation of behaviour in the selection of land uses and activities respectively, and their accompanying transition rules. In local agent based models, individual differences can be studied since each agent has his own characteristics, including his own believes desires and intentions, while the other approaches typically include individual differences only in space and in some stochastic variation that is added to the agent behaviour. The global agent based approach does not include individual differences, as the focus is on the result of group behaviour.

Table 1. Overview of characteristics of the various categories of agent based land use change models ${ }^{1}$.

\begin{tabular}{|l|l|l|l|l|l|}
\hline & $\begin{array}{l}\text { Constrained } \\
\text { CA }\end{array}$ & $\begin{array}{l}\text { Constrained } \\
\text { activity based }\end{array}$ & $\begin{array}{l}\text { Cellular } \\
\text { agents }\end{array}$ & $\begin{array}{l}\text { Global agent } \\
\text { based }\end{array}$ & $\begin{array}{l}\text { Local agent } \\
\text { based }\end{array}$ \\
\hline Decisions taken by & Land use type & Activity type & Cell & $\begin{array}{l}\text { Individual } \\
\text { agent }\end{array}$ \\
\hline Land use competition & Local & Local & Local & Global & Local \\
\hline $\begin{array}{l}\text { Interaction of agent } \\
\text { with the landscape }\end{array}$ & Local & Local & Local & Top down & Local \\
\hline $\begin{array}{l}\text { Agent heterogeneity } \\
\text { \& stochastic } \\
\text { variation }\end{array}$ & $\begin{array}{l}\text { A stochastic } \\
\text { variation }\end{array}$ & $\begin{array}{l}\text { Agent types } \\
\text { differences }\end{array}$ & Agent types & $\begin{array}{l}\text { Agent types } \\
\text { individual } \\
\text { differences }\end{array}$ \\
\hline Data demands & Low & Medium & High & Medium & High \\
\hline Applicable area extent & $\begin{array}{l}\text { Cities to } \\
\text { continental }\end{array}$ & $\begin{array}{l}\text { Cities to } \\
\text { countries }\end{array}$ & $\begin{array}{l}\text { Small regions } \\
\text { and cities }\end{array}$ & $\begin{array}{l}\text { Small regions } \\
\text { and cities }\end{array}$ & $\begin{array}{l}\text { Small regions } \\
\text { and cities }\end{array}$ \\
\hline $\begin{array}{l}\text { Computational } \\
\text { requirements }\end{array}$ & Low & Medium & Medium & Low & High \\
\hline Process accuracy & Low & Medium & Medium/high & Medium/high & High \\
\hline Predictive accuracy & Medium & Medium/low & Low & Medium/low & Low \\
\hline $\begin{array}{l}\text { Proven track record in } \\
\text { spatial planning }\end{array}$ & Yes & No & No & No & No \\
\hline
\end{tabular}

Due to their high data demands and computation requirements we find that agent based models with local agents and cellular agents are mostly applied at small scales, while CA applications can be found from the local to the continental scale. Activity based models have been found from the local to the national scale, which seems understandable given that their data requirements are slightly higher than those of CA models (the approach requiring data on activities per cell). The applicable area extent of global agent based models seems to depend mainly on the choice of the agent groups. Because global agents are representative for a group of actors in the entire area, the application domain for these models is typically smaller regions.

We found that there seems to be a trade-off between the process accuracy and the predictive accuracy (Brown et al, 2005): the more detail is included in the process description, the better it resembles the actual process, but the higher the data requirements, the harder the calibration and the fewer the predictive

\footnotetext{
${ }^{1}$ We have defined characteristics for the five categories based on existing models. This means that some limitations that were found are not necessarily a limitation of the approach, but rather of the current available models.
} 
capabilities. It is most likely for this reason that process understanding is often mentioned as the main aim of the local agent based approach, while CA models are found as part of SDSS and have a proven track record in spatial planning.

\section{CONCLUSIONS}

Based on a literature review we have grouped agent based land use change models into five categories according to the way in which human behaviour is represented in them. We found that all five types incorporate human behaviour and human decision making in some way, but that the degree to which they do and the detail with which they do so varies considerably. At the same time we found that detail in agent representation generally comes at the cost of data and computation requirements as well as an increase in uncertainty and hence there is a trade-off between process and predictive accuracy. We conclude that there is not one best way to include agents in spatial decision support systems, but that instead this decision is a trade-off between the different properties related to each of the five types. The decision on the type of agent behaviour to be incorporated in a spatial decision support system highly depends on data availability and area extent, but also on the type of information and practical considerations required by the (potential) users.

\section{ACKNOWLEDGMENTS}

This categorisation is made in the context of the Sustainable Pathways project (http://www.sp2.org.nz).

\section{REFERENCES}

Brown, D.G., Page, S., Riolo, R., Zellner, M., Rand, W., 2005. Path dependence and the validation of agentbased spatial models of land use. Int. Journal of Geographical Information Systems 19 (2): 153-174.

Clarke, K.C., Hoppen, S., Gaydos, L.J., 1998. A self modifying cellular automaton model of historical urbanization in the San Fransisco Bay area. Environment and Planning B 24: 247-261.

De Nijs, T.C.M., de Niet, R., Crommentuijen, L., 2004. Constructing land-use maps of the Netherlands in 2030. Journal of Environmental Management 72: 35-42.

Dean, J.S., Gumerman, G.J., Epstein, J.M., Axtell, R.L., Swedlund A.C., Parker, M.T., McCarroll, S., 2000. Understanding Anasazi culture change through agent-based modeling. In: Kohler, T.A., Gumerman, G.J. Dynamics in Human and Primate societies: Agent-based modeling of social and spatial processes. New York, Oxford university press.

Haase, D., Lautenbach, S., Seppelt, R., 2010. Modeling and simulating residential mobility in a shrinking city using an agent-based approach. Environmental Modelling and Software 25: 1225-1240.

Kok, K., Van Delden, H. 2009. Combining two approaches of integrated scenario development to combat desertification in the Guadalentín watershed, Spain, Environment and Planning B 36: 49-66.

Ligtenberg, A., Bregt, A.K., Van Lammeren, R.J.A., 2001. Multi-actor-based land use modelling: spatial planning using agents. Landscape and Urban Planning 56: 21-33.

Matthews, R.B., Gilbert, N.G., Roach, A., Polhill, J.G., Gotts, N.M., 2007. Agent-based land-use models: a review of applications. Landscape Ecology 22: 1447-1459.

Parker, D.C., Manson, S.M., Janssen, M.A., Hoffman, M.J., Deadman, P., 2003. Multi-Agent systems for the simulation of land use and land cover change: A review. Annals of the association of American Geographers 92(2): 314-337.

RIKS, 2010. Metronamica - Model descriptions. RIKS, Maastricht, The Netherlands: www.metronamica.nl

Robinson, D.T., Brown, D.G., Parker, D.C., Schreinemachers, P., Janssen, M.A., Huigens, M., Wittmer, H., Gotts, N., Promburom, P., Irwin, E., Berger, T., Batzweiler, F., Barnaud, C., 2007. Comparison of empirical methods for building agent-based models in land use science. Journal of Land Use Science 2(1): 31-55.

Sieber, S., Zander, P., Verburg, P.H., Van Ittersum, M., 2010. Model-based systems to support impact assessment - Methods, tools and applications. Ecological Modelling 221(18): 2133-2135.

Smajgl, A., 2010. Challenging beliefs through multi-level participatory modelling in Indonesia. Environmental Modelling and Software 25(11): 1470-1476.

Van Delden, H., Luja, P., Engelen, G., 2007. Integration of multi-scale dynamic spatial models of socioeconomic and physical processes for river basin management. Environmental Modelling and Software 22(2): 223-238.

Van Vliet, J., Hurkens, J., White, R., Van Delden, H., (in press). An activity based cellular automaton model to simulate land use dynamics. Environment and Planning B.

Yeh, A.G.O., Li, X., 2002. A cellular automata model to simulate development density for urban planning. Environment and Planning B 29: 431-450. 